\title{
A Method of Logistics Information Security Based on Blockchain Technology
}

\author{
Haiman Liu $^{1^{*}}$, Ruoying Sun ${ }^{1}$, Gang Zhao ${ }^{1}$ \\ 1. School of Information Management, Beijing Information Science and Technology University \\ Beijing, China \\ lanny1212@163.com, sunry@bistu.edu.cn, zhaogang@bistu.edu.cn
}

\begin{abstract}
With the development of E-commerce, the logistics industry develops rapidly at the same time. The negative influence lead by logistics information security can not be ignored. In this paper, we proposed a method of logistics information security based on blockchain technology to handle the issues of logistics information fraud and defective supervision mechanism. After comparing the typical logistics business process with logistics business process based on blockchain technology, we designed the smart contract of logistics information security. In the experiment, a normal use case and two abnormal use cases are introduced to show the processes of smart contract. The results show that the designed smart contract can effectively ensure the logistics information security.
\end{abstract}

Keywords-logistics information security; blockchain; smart contract; E-commerce

\section{INTRODUCTION}

With social progress and development of technology, Ecommerce develops rapidly, which further promote logistics industry at the same time, especially express delivery business. The report shows that the scale of express delivery business of Chinese has situated at first in the world continuously for four years, which will account for $40 \%$ of the global market by then[1]. Convenient and effective express delivery business can support the development of E-commerce effectively, but the issues of logistics information fraud and defective supervision mechanism can't be ignored, which would lead to negative influence.

Taking the express delivery business in E-commerce as an example, participants mainly include E-commerce platform, consignor, carrier, consignee, third party payment and bank in the whole process. The whole E-commerce and logistics information mainly provided by carrier in express delivery business and stored in the information system of E-commerce platform and carrier, which means consignee or normal customer can only passive receive logistics information with low participation. When meeting unfair or illegal situation, such as logistics information fraud and defective supervision mechanism, customers has no choose but only appeal to the platform to protect their interests. At the same time, records in the whole trading and logistics process rely on manual input and handing. It means slow processing speed and low treatment efficiency. For instance, as shown in figure 1, from the information of a bought product in Tmall, an E-commerce platform of Alibaba, we can clearly find out the product was bought in August 23th, 2018, but the logistics information shows that the product has been signed for in February 21th, 2018, half year ago. The obvious fake logistics information didn't trigger any exception or hint in e-commerce platform. If the logistics information security can't be ensured, the ecommerce platform, express delivery company and customer would be discouraged.

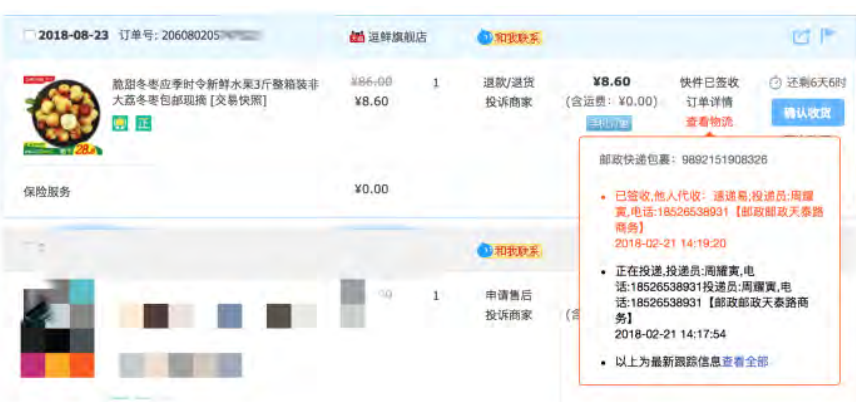

Fig. 1 Example of Fake Logistics information

Aiming at the situation, this paper proposed a method of logistics information security based on blockchain technology by utilizing smart contract to normalize and restrain the behaviors of all participants in the logistics and trading process, thus ensure logistics information security. In experiments, we use solidity to develop a testing smart contract in Ethereum and test typical logistics and trading use cases. The results show that the proposed method based on blockchain technology can effectively mitigate the risk in the logistics and trading process.

The chief contributions of our work are summarized as follows:

(1) To our best of our knowledge, this is the first work that proposing a method based on blockchain technology to handle the issue of logistics information security, including fake logistics information, fast exception handing, automatic information processing and so on.

(2) Aiming at typical logistics and trading cases, we designed testing use cases. These use cases can effectively evaluate the completeness, reliability and validity of smart contract. 
In addition to this introductory, the remainder of our paper is organized as follows. Section 2 summarizes related work in logistic information security ad blockchain technology. In Section 3, we introduce the research ideas and solution. Next, Section 4 shows the processes and results of experiment. Finally, we present out conclusions and avenues for future work in Section 5.

\section{RELATED WORK}

Research about logistics security mainly includes safe transportation mechanism for special articles, analysis of safe delivery model, logistics traceback and so on. Among them, in the aspect of transportation mechanism for special articles, Xueyu Mi[2] did research on safe transportation and management mechanism of explosives for use at mines by developing information system to optimize warehousing, storage operation, driven route and vehicle localization. Fuchang Li[3] took Yunnan matsutake as research object to design the optimization scheme of logistics distribution routine problem. Optimizing measures include carrying out joint distribution mode, building safe and reliable cold chain information sharing platform, setting distribution center and so on. Zhongwei $\mathrm{He}[4]$ discussed and analyzed logistics distribution of fresh agricultural product in development trend and quality safety. In aspects of safe distribution model, Weidong Li[5] found out some safety problems in distribution process, including cooperation risk within enterprises, risk of product distribution and infrastructure, risk of distribution process and product value, risk of uncontrolled external environment and so on, which would affect the development of logistics industry. The author come up with building a safety model in supply chain management to ensure that distribution process is effectively. In aspect of logistics traceback, Minmin Xiao[6] studied using RFID technology to achieve long-term supervision and traceback mechanism.

From the above, the current researches on logistic safety or security mainly focused on human factor and management factor. By using kinds of technologies and methods to ensure logistics safety or security in each stage. Technology of block chain is well to solve these problems facing in logistics, so industry paid full attention.

As the concept of Internet plus has been proposed, block chain technology gradually from hype to practical application. In October 2016, China's Ministry of Industry and Information Technology released "China Blockchain Technology and Application Development White Paper"[7] and pointed that the applications of blockchain had extend to Internet of Thing, intelligent manufacturing, supply chain management and so on. As the description of Blockchain by Wikipedia[8], Blockchain is a growing list of records, called blocks, which are linked using cryptography. Each block contains a cryptographic hash of the previous block, a timestamp, and transaction data. Blockchain was invented by Satoshi Nakamoto in 2008 to serve as the public transaction ledger of the cryptocurrency bitcoin[9]. Gartner[10] also listed blockchain technology as the 2016 Hype Cycle for Emerging Technologies and holds the idea that there are five years to ten years before blockchain technology being adopted by mainstream. Except mature application in financial services industry so far, applications of blockchain technology in other industries are at the beginning and still under study[11].

Till now, there is some researches about using blockchain technology in logistics business. Center for Transportation and Logistics, MIT hold a roundtable to define blockchain system and explore the benefits that blockchains might deliver and how the technology could be applied today and future[12]. K Sadouskaya[13] analyzed the innovative technology blockchain and the potential of blockchain-based application and interviewed to find out possible challenges or benefits of blockchain based application. Fredrik Jansson[14] develop a framework for evaluating the applicability of blockchain technology in supply chain management to improve traceability. Amina Badzar[15] focused on increasing supply chain transparency for both suppliers and consumer, and improving contractual coordination in order to secure fulfilment of sustainability clauses in transportation contracts. Niels Hackius[16] conducted an online survey and asked logistics professionals for their opinion on use case exemplars, barriers, facilitators, and the general prospects of blockchain in logistics and supply chain management. Results shows that participants are fairly positive about this new technology and the benefits it offers.

In summary, based on the idea of decentralization and the features of inline validation, traceability, hard to tamper with and unable to block, blockchain technology can be a good alternative scheme to existing logistics business information system. It is significant to use blockchain technology in logistics business, especially logistics information.

\section{RESEARCH IDEAS AND SOLUTION}

In order to handle the issues of logistics information security, blockchain technology can be employed to ensure logistics security in each stage. We compared logistical business process based on blockchain technology with typical logistical business processes to show our research ideas. Then we introduced design of smart contract to show the solution. Detail introductions are shown as follows.

\section{A. Scheme Comparison}

In Figure 2, we compared logistical business process based on blockchain technology with typical logistical business processes. The current typical logistical business process is shown in Figure 2.1. Roles include E-commerce platform, consignor, carrier, third party payment, bank and consignee. By bring in third party payment, the security of typical scheme in logistics and trading process can be assured. Data generated in the whole process would be stored in the database of Ecommerce platform, consignor and carrier. Considering the technical capacity and business motivation, there are risks of data corruption, tampering and falsification. For example, if the consignor doesn't send the package off, he also can update the status to "delivered". There is no mechanism to verify and constraint the behavior information. At the same time, the transaction security relies on third party payment, which would increase the complexity and cost. 

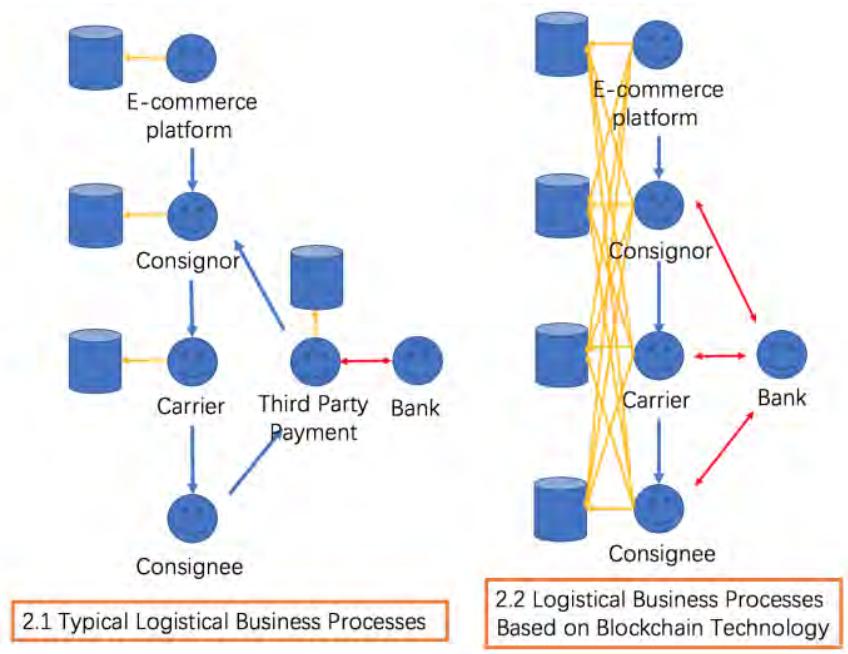

Fig. 2 Scheme Comparison

Figure 2.2 shows logistical business processes based on blockchain technology. By using blockchain technology, data would be stored in distributed databases of all participants. Data can only be added, can't be deleted and modified, which means fake information would be recorded forever. At the same time, by utilizing smart contract to control the whole business process, when all business start, work and finish by setting rules, the whole operations would be finished automatically and do not need third party payment. In this scheme, logistics information security can be ensured and trading efficiency would be improved.

\section{B. Design of Smart Contract}

In design process of smart contract, security principles of Zero Trust would be adopted. The identity and authority of all participants need to be affirmed. When the logistic order generated and started, all behaviors of rules in each stage would be restrict. The constraints would be defined in smart contract when initializing. Typical constraints include time constraint and conditional constraint. Time constraint dictates how long consignor must set out the goods, the suggested logistics time on the way, default time of automatic receive and other restraints. Conditional constraint includes can't adding data after the order is closed and so on.

In normal process, operations of each roles need to meet the defined specifications or constraints. After the logistics order is closed, the roles who achieved the requirements will get corresponding reward in reputation. Roles with good reputation will get more trust, and then enjoy better services or make more money.

Abnormal process may be trigger by error permission, abnormal operations of each role, and illegal process logic. In abnormal process, all exception would be handled in an automated way, including automated deducting reputation score, automated ignoring the exception or automated rollback operation and handling the property. If the exception can be accepted, such as carriers' delivery delay or lost the package, after the order is closed, related responsible participants would be deducted reputation score. If the exception can't be accepted, the whole process would be rollback automatically and related responsible participants would be punished. If the process has rollback to the consignor, it means the order has failed. The order money will return to customer and the order be closed.

The designed code and logic are shown as follows.

contract Logistics\}

// All participants in logistics process

struct Role\{\}

// Basic order information

struct Order\{\}

// Simple logistics information

struct LogisticsInfo\{\}

// Init logistics order information

function initLogistics\{\}

// Init identity and authority

function initRole\{\}

// Consignor send package

function sendPackage\{\}

// Carrier collect package

function collectPackage\{\}

// Carrier transport package

function transportPackage \{\}

// Carrier deliver package

function deliverPackage \{\}

// Consignee receive package

function receivePackage \{\}

// Judge when abnormal behavior

function judge \{\}

//Award when finish as expect 
function award \{\}

\section{// Rollback when trigger particular conditions}

function rollback \{\}

// View the record of logistics

function viewRecord \{\}

// Automatic transfer money when order accomplish

function payment\{\}

l

According to Zero Trust principle[17], when designing the smart contract, all roles' identity needs to be verified. Smart contract does not trust any host and IP address. Only the authorized roles can call the function of smart contract to add or read logistics data information. Thus, the smart contract can ensure all data is true in the whole logistics business process and will be recorded into blockchain system.

\section{EXPERIMENT}

In experiment, we use normal use cases and abnormal use cases to test the smart contract's function. In normal use cases test, we mainly test data superaddition and smart contract automatic execution. In abnormal use cases test, several abnormal conditions need to be considered, including show as "sent out goods" but do not notice carries pick-up, wrong logistic information time, receiving damaged package, package lost on the way, consignee forget to confirm reception and so on. The typical tests are shown as follows.

In normal use cases test, steps are as follows:

(1) Calling function initRole \{\} to check the authority of all participants.

(2) E-commerce platform call function initLogistics \{\} to add order information and logistics information into blockchain databases.

(3) Consignor call function sendPackage \{\} to inform carrier collect package.

(4) Carrier call function collectPackage \{\} to collect package.

(5) Carrier call function transportPackage \{\} when transport package in each step.

(6) Carrier call function deliverPackage \{\} to inform consignee receive package.

(7) Consignee call function receivePackage \{\} when received package.

(8) Smart contract automatic call function payment \{\} to transfer consignee's money to consignor's account.
(9) Smart contract automatic call function award \{\} . According to the logistic information in each step to add the reputation score to corresponding participants.

In abnormal use cases: show as "sent out goods" but do not notice carries pick-up, steps are as follows:

(1) Calling function initRole \{\} to check the authority of all participants.

(2) E-commerce platform call function initLogistics \{\} to add order information and logistics information into blockchain databases.

(3) Consignor call function sendPackage \{\} but do not inform carrier collect package in time.

(4) Smart contract detect abnormal behavior, call function judge() to judge the issue and deduct reputation score of consignor.

(5) Smart contract call function rollback() to send back the logistics information to consignor.

In abnormal use cases: package lost on the way, steps are as follows:

(1) Calling function initRole \{\} to check the authority of all participants.

(2) E-commerce platform call function initLogistics \{\} to add order information and logistics information into blockchain databases.

(3) Consignor call function sendPackage \{\} to inform carrier collect package.

(4) Carrier call function collectPackage \{\} to collect package.

(5) Carrier call function transportPackage \{\} when transport package in each step.

(6) Smart contract detects that carrier do not call function deliverPackage() out of time, or carrier add lost package information by himself, call function judge() to judge by constraint condition defined in order information.

(7) According the judging result, smart contract decide to call function rollback or terminates the process.

\section{CONCLUSIONS}

In this paper, we proposed a method of logistics information security based on blockchain technology to hand the issues of logistics information fraud and defective supervision mechanism. By comparing the typical logistics business process with logistics business process based on blockchain technology, the advantage of blockchain appeared. Based on the process, the smart contract was designed and introduced. In experiment, a normal use case and two abnormal use cases was introduced to show the processes of smart contract. The results show that the designed smart contract can effectively ensure the logistics information security.

Considering that the model used in this paper has been simplified in a degree, there are still lots of works to use the 
scheme in real business. Besides, performance also is a key factor when deployed in actual scene. These are the significant content that need to be considered in the future.

\section{ACKNOWLEDGMENT}

This work was supported by the Major Program of the National Social Science Foundation of China (Grant No.14ZDB133) and the Natural Science Foundation of China under Grant No.615720279.

\section{REFERENCES}

[1] [1] China Daily, China's express delivery business ranks first in the world for four consecutive years[EB/OL], http://caijing.chinadaily.com.cn/2018-02/27/content_35750441.htm, 2018

[2] [2] Xueyu Mi, Research on Technical Framework of Mine Explosives Storage and Distribution Safety Management System[J], 2011.

[3] [3] Yidan Zhang, Optimization of Inventory Strategy of Yunnan Pine Mushroom Cold Chain Logistics Based on Safety and Reliability[J], Resource development and market, 2014.

[4] [4] Zhongwei He, Development Trend and Quality Safety of Beijing Fresh Agricultural Products Logistics Distribution Industry[J], Beijing Social Sciences, 2010

[5] [5] Weidong Li, Analysis of Distribution Security Mode in Supply Chain Management $[J]$, Consumer Electronics, 2013.

[6] [6] Minmin Xiao, Research on Traceability System of Food Cold Chain Logistics Based on RFID[D], Beijing Jiaotong University, 2012.
[7] [7] Ping Zhou, China Blockchain Technology and Application Development White Paper[O], Beijing: Ministry of Industry and Information Technology, 2016.

[8] [8] Blockchain[EB/OL], https://en.wikipedia.org/wiki/Blockchain.

[9] [9] Nakamoto S. Bitcoin: A peer-to-peer electronic cash system[J]. 2008.

[10] [11] Gartner, Gartner's 2016 Hype Cycle for Emerging Technologies Identifies Three Key Trends That Organizations Must Track to Gain Competitive Advantage[EB/OL] https://www.gartner.com/newsroom/id/3412017, 2016

[11] [12]Xiaohua Zhang, Analysis of Blockchain Technology and Its Application in Logistics[J], Science and technology economic guide, 2017.

[12] [13] Blockchain in Supply Chains: Looking Beyond the Hype, https://ctl.mit.edu/events/tue-10242017-1730/blockchain-supply-chainslooking-beyond-hype, 2017.

[13] [14] Sadouskaya K. Adoption of Blockchain Technologyin Supply Chain and Logistics[J]. 2017.

[14] [15] Petersen O, Jansson F. Blockchain Technology in Supply Chain Traceability Systems[J]. 2017.

[15] [16] Badzar A. Blockchain for securing sustainable transport contracts and supply chain transparency-An explorative study of blockchain technology in logistics[J]. 2016.

[16] [17] Hackius N, Petersen M. Blockchain in logistics and supply chain: trick or treat?[C]. epubli, 2017.

[17] [18] Paloalto Networks, WHAT IS A ZERO TRUST ARCHITECTURE? [EB/OL], https://www.paloaltonetworks.com/cyberpedia/what-is-a-zerotrust-architecture. 